La Revue

des Droits

de l'Homme

\section{La Revue des droits de l'homme}

Revue du Centre de recherches et d'études sur les droits fondamentaux

$17 \mid 2020$

Revue des droits de l'homme - $\mathrm{N}^{\circ} 17$

À propos de Lynne SEGAL, Out of Time. The Pleasures \& Perils of Ageing. Préface d'Elaine Showalter, Verso, London, New York, 334 p., 2013.

\title{
Explorations féministes de l'avancée en âge
}

Juliette Rennes

\section{(2) OpenEdition}

\section{Journals}

Édition électronique

URL : http://journals.openedition.org/revdh/8827

DOI : $10.4000 /$ revdh.8827

ISSN : 2264-119X

Éditeur

Centre de recherches et d'études sur les droits fondamentaux

Référence électronique

Juliette Rennes, «Explorations féministes de l'avancée en âge », La Revue des droits de l'homme [En ligne], 17 | 2020, mis en ligne le 24 février 2020, consulté le 19 octobre 2020. URL : http:// journals.openedition.org/revdh/8827 ; DOI : https://doi.org/10.4000/revdh.8827

Ce document a été généré automatiquement le 19 octobre 2020

Tous droits réservés 

2013.

\title{
Explorations féministes de l'avancée en âge
}

\author{
Juliette Rennes
}

\section{I. Âge, genre, génération et rapports de domination}

En novembre 2019, lors d'une session parlementaire en Nouvelle-Zélande, la députée Chloe Swarbrick, vingt-cinq ans, évoque l'urgence d'agir contre le changement climatique. Interrompue par un député plus âgé, elle se tourne vers lui et lui rétorque calmement un «OK boomer » avant de reprendre, comme si de rien n'était, son propos. Si cette expression existait avant novembre 2019, la médiatisation de cet échange parlementaire a contribué à la large diffusion du «OK Boomer». Face à des personnes occupant des positions de pouvoir, qui invoquent leur savoir et leur expérience pour discréditer les aspirations de transformation sociale de personnes plus jeunes, « $O K$ boomer " peut sonner comme une résistance légitime à des relations de domination indissociablement liées à la différence d'âge, de statut socioéconomique et souvent de genre $^{1}$. Cependant, tel qu'elle est utilisée, cette expression tend souvent à réduire les opinions conservatrices à un simple effet de génération indépendamment des autres dimensions des rapports sociaux. Pour mettre en lumière le succès de "OK boomer", nombre de médias français n'ont d'ailleurs pas manqué de publier des dossiers spéciaux sur le «fossé des générations » en reléguant à l'arrière-plan les clivages politiques intragénérationnels et les héritages militants intergénérationnels, depuis les années 1970, y compris en matière d'écologie. Et si le "OK boomer » peut être perçu comme un geste d'émancipation lorsqu'il met en question l'arrogance de ceux - et dans une moindre mesure de celles - qui occupent des positions de pouvoir, s'en prendre à la génération du baby-boom peut tout aussi bien s'inscrire dans une rhétorique beaucoup moins subversive consistant à accuser les "boomers" (et en particulier les «soixante- 
huitards ») de tous les maux, et notamment celles et ceux qui constituent aujourd'hui une partie importante des retraités, de représenter un fardeau insurmontable pour les finances publiques et le reste de la population. De part et d'autre de l'Atlantique, ce discours, souvent inscrit dans un agenda politique anti-État social, est décortiqué de façon critique dans plusieurs travaux de chercheuses et de chercheurs sur l'âgisme dénonçant le «boomer bashing ${ }^{2}$ ».

2 C'est aussi avec cette question que s'ouvre l'essai Out of Time, publié en 2013 par Lynne Segal, professeure de psychologie et d'études de genre au Birbeck College à Londres. Son analyse des usages idéologiques des «clivages générationnels » constitue le point de départ d'une réflexion plus large sur les relations entre générations ainsi que sur l'expérience et la perception du vieillissement - entendu comme processus indissociablement social et biologique de transformation corporelle et psychique lié à l'avancée en âge - et sur la vieillesse, entendue comme « dernière période » du parcours de vie, dont le seuil varie selon les époques et les sociétés, mais aussi selon le genre et le statut social. Lynne Segal enquête sur ces questions en mobilisant les études sur le genre et explore plus largement ce que pourrait être une approche féministe du vieillissement et de la vieillesse. Une telle perspective de recherche n'est certes pas nouvelle : depuis les années 1980, un nombre conséquent d'ouvrages, notamment en langue anglaise, ont analysé le vieillissement à partir d'un questionnement féministe ${ }^{3}$, tandis que depuis les années 2010 , un champ des «age studies » tend à se développer dans le sillage des études de genre ${ }^{4}$. Dans ce contexte académique, l'originalité de l'ouvrage de Segal ne tient pas tant au défrichage d'un nouveau champ d'études, qu'à sa démarche d'enquête: Out of Time est une réflexion à partir de son expérience personnelle, informée à la fois par ses recherches antérieures et par une large compilation de travaux psychanalytiques, sociologiques, historiques, littéraires et poétiques sur le vieillissement et la vieillesse. Explicitant sa situation au moment où elle écrit, celle d'une femme vivant en Grande-Bretagne, «babyboomeuse ", universitaire, féministe, engagée à gauche, qui approche des 70 ans au moment où elle écrit ce livre (elle est née en 1944), c'est à partir de cette position située qu'elle explore l'expérience de vieillir, le regard social sur la vieillesse et les relations entre générations.

\section{La vie mentale dans les corps âgés}

3 À partir de ce matériau d'enquête diversifié, l'objet du livre, résume Segal, est d'essayer de rendre compte des "complexités de la vie mentale dans les corps âgés ${ }^{5}$. Et c'est d'abord pour tenter de domestiquer ses propres craintes et préjugés face à la vieillesse qu'elle a ressenti le besoin d'enquêter sur la façon dont d'autres avant elles - surtout des femmes, mais aussi quelques hommes - l'avaient expérimentée et mise en mots: "Quel âge ai-je? Ne demandez pas, ne dites rien. La question me fait peur. Elle est exaspérante, d'autant plus pour celles qui, comme moi, féministes de gauche [...] se targuent d'avoir consacré tant de temps à combattre les préjugés de toutes sortes ».

4 Cette ambivalence, elle la rencontre dans d'autres écrits de femmes, à commencer par ceux de Simone de Beauvoir, dont un portrait illustre la couverture de Out of Time. Dans Le Deuxième Sexe, paru en 1949, Beauvoir analyse de façon percutante le regard social particulièrement négatif sur le vieillissement féminin comme une dimension des inégalités de genre ${ }^{6}$. Pourtant, à la fin du volume de son autobiographie La Force des 
choses en 1963, alors qu'elle n'a que 55 ans, elle confie le dégoût qu'elle éprouve face à son propre corps vieillissant en présentant comme inévitables et naturels ces affects négatifs. Elle dépeint également toute une série de renoncements qui, selon elle, l'attendent fatalement dans les années à venir7. En réalité, quelques années après, sa rencontre avec Sylvie Le Bon qui deviendra sa fille adoptive et son engagement enthousiaste dans le mouvement féministe infirmeront en partie ses prédictions négatives. Cependant, son livre La Vieillesse paru en 1970 présente, lui aussi, certaines contradictions : alors qu'elle y dénonce lucidement l'altérisation et l'infériorisation sociale des personnes catégorisées comme vieilles, elle distingue elle-même constamment les "adultes" et les "personnes âgées", n'échappant pas à cette représentation des vieilles et des vieux comme incarnant une forme d'altérité radicale ${ }^{8}$. Pour Segal, il s'agit alors de prolonger le double geste initié par Beauvoir, de dévoilement des dimensions socialement construites à la fois de la vieillesse et du genre, tout en allant plus loin dans le travail ouvert par Beauvoir pour imaginer une forme de vieillissement et de vieillesse qui pourrait changer l'expérience subjective qu'en ont les femmes et les hommes.

\section{Féminisme des années 1970 et anti-âgisme}

5 Le féminisme, note Segal, peut sembler une bonne armure pour résister à l'âgisme. En effet, l'une des manifestations de l'âgisme qui touche davantage les femmes que les hommes réside dans la disqualification des marques corporelles visibles de l'avancée en âge, assimilées à une dégradation d'un modèle de beauté jeune. Or les militantes féministes des années 1970 se sont précisément efforcées de déconstruire le regard masculin (« male gaze ») focalisé sur la figure de la jeune femme considérée comme seul étalon de beauté féminine. On trouve ainsi quelques textes percutants, dans les années 1970, analysant cette hiérarchisation entre femmes jeunes et vieilles comme une composante de la domination masculine, une hiérarchisation qui n'existe pas de manière symétrique entre jeunes hommes et hommes mûrs ${ }^{9}$. Pourtant, le mouvement féministe de ces mêmes années ne s'est pas entièrement émancipé du regard âgiste sur les femmes plus âgées, rappelle Segal : des féministes qui avaient alors la quarantaine ou la cinquantaine, quand la plupart des militantes avaient la vingtaine, étaient regardées par les plus jeunes avec mépris, n'étaient pas invitées aux groupes de parole, ou étaient explicitement renvoyées à leur appartenance générationnelle, celle d'une " génération traître ", qui se serait repliée dans les années 1940-1960 sur la maternité et la famille. C'est ce dont témoigne, parmi d'autres, la sociologue féministe Meg Stacey, 50 ans en 1972, qui écrit avoir souffert de cette stigmatisation fondée sur son âge et son appartenance générationnelle ${ }^{10}$.

6 Contre la reconduction indéfinie de ce clivage entre femmes jeunes et vieilles qui s'immisce jusque dans le mouvement féministe qui aurait dû le déconstruire, Segal appelle de ses vœux la constitution d'un « nous ", sujet des luttes féministes, qui ne soit pas implicitement borné par des frontières d'âge, ce qui implique en vieillissant d'être " capable de conserver certains liens et affiliations à travers les générations, sans trop d'envie, d'amertume, de sentiment d'exclusion et de perte». Cette construction d'un mouvement féministe transgénérationnel implique aussi - ce qui demeure dans l'ombre de cet ouvrage - un travail des femmes plus âgées pour lutter contre la tentation de disqualifier la parole et les projets des générations plus jeunes, en les renvoyant à leur 
âge et leur inexpérience, comme on l'évoquait en ouverture de cet article. Depuis le début des années 1990, toute une littérature féministe porte sur les difficultés à forger des collectifs féministes à la fois horizontaux - la fameuse «sororité»- et intergénérationnels. Le besoin de se retrouver entre pairs en âge est susceptible de produire des effets âgistes, mais il peut aussi témoigner d'une aspiration égalitaire, celle d'expérimenter des rapports émancipés de relations de domination fondées sur l'écart d'âge ${ }^{11}$. Un défi qui est encore d'actualité pour les luttes féministes - mais aussi pour toute mobilisation - serait donc précisément de fabriquer de l'égalité et de l'horizontalité sans procéder à des exclusions fondées sur des critères d'âge et de génération, de faire coexister au sein d'une lutte à la fois des espaces d'entre-soi intragénérationnels et des espaces collectifs intergénérationnels. Surmonter l'âgisme permet aussi d'éviter l'ignorance néfaste des luttes menées par les générations plus âgées, comme l'écrivait la poétesse et militante féministe noire américaine Audre Lorde (1934-1992) : "Le "fossé des générations" est un outil de toute société répressive. Si les jeunes membres d'une communauté considèrent les plus âgés comme méprisables, suspects ou superflus, ils ne pourront jamais se donner la main et examiner les souvenirs vivants de la communauté, ni poser la question importante : "Pourquoi ?" Cette amnésie historique nous conduit constamment à réinventer la roue ${ }^{12}$.

\section{Les « ambigüités radicales de la vieillesse » : genre et vieillissement}

7 Passant d'une analyse de l'appartenance générationnelle à celle de l'expérience de vieillir, Segal s'intéresse aux récits autobiographiques contemporains qui problématisent cette expérience. L'autrice note les sentiments très contradictoires face à la vieillesse exprimés dans ces récits à la première personne : angoisse, mélancolie et sensation d'abattement peuvent coexister avec un sentiment de renouveau, d'émancipation et de réinvention de soi ; l'expérience de la perte de son ancien " moi » peut s'entremêler avec celle de la continuité de soi : vieillir, c'est aussi porter avec soi toutes les strates de ses «moi » passés, voir resurgir parfois des plaisirs et des douleurs de l'enfance ${ }^{13}$. Ces ambivalences sont en partie communes à l'expérience des femmes et des hommes, mais Lynne Segal explore ce que ces récits autobiographiques nous disent des différences de genre. Même si elle fait également, à de nombreuses reprises, référence aux différences de classe face au vieillissement et à la vieillesse, elle n'utilise pas directement ses principaux matériaux d'enquête (sa propre expérience et des récits autobiographiques publiés par des écrivains, des universitaires et des artistes qui ont de fait des statuts socioéconomiques peu diversifiés) pour éclairer la question des inégalités socioéconomiques dans l'expérience subjective de vieillir.

Concernant les différences de genre, note Lynne Segal, on peut penser d'un côté que les femmes ont davantage à perdre en vieillissant que les hommes puisqu'on juge plus sévèrement les marques corporelles de leur avancée en âge non seulement dans les relations intimes, mais aussi dans le monde professionnel (Segal cite plusieurs enquêtes sur les comédiennes, mises à la retraite avant les hommes); les femmes âgées sont plus souvent célibataires et leurs ressources économiques en tant que retraitées sont en moyenne très inférieures à celles des hommes retraités. Pourtant, remarque l'autrice, nombre de travaux - par exemple sur la « sursuicidité » masculine qui existe à tous les âges, mais s'accentue très fortement aux âges élevés - suggèrent à l'inverse que sous un 
certain angle, il est plus difficile pour les hommes de vieillir que pour les femmes. Simone de Beauvoir elle-même dressait ce constat dans La Vieillesse: analysant différents corpus d'entretiens avec des femmes et des hommes retraités, elle concluait que les hommes tendaient à perdre davantage que les femmes le sentiment de leur utilité sociale parce que la société les incitait à s'identifier entièrement à leur statut professionnel et à leur rôle de «monsieur gagne-pain » alors que les femmes faisaient davantage l'expérience d'une continuité avec leur vie passée, notamment à travers l'engagement dans des activités domestiques et des relations affectives avec des membres de la famille.

Dit autrement, la vieillesse constituerait une mise à l'épreuve périlleuse des normes de la virilité. Selon Segal, nombre d'œuvres littéraires et autobiographiques contemporaines écrites par des hommes - notamment Philippe Roth, Martin Amis ou John Updike - sont particulièrement pessimistes sur la vieillesse : les ouvrages dont elle rend compte suggèrent que cette période de la vie fait voler en éclats le sentiment d'invulnérabilité que les hommes, davantage que les femmes, sont incités à éprouver au cours de leur vie adulte; la définition de la sexualité masculine comme pouvoir se trouve en outre menacée par "l'impuissance", une métaphore significative pour désigner les difficultés érectiles, thème récurrent des ouvrages dont Segal rend compte. S'appuyant sur la lecture d'enquêtes sur la sexualité et notamment celle de la sociologue Linn Sandberg auprès d'hommes hétérosexuels de plus de $70 \mathrm{ans}^{14}$, Segal suggère que les transformations corporelles liées à l'avancée en âge pourraient justement amener des hommes à remettre en cause les conceptions « phalliques » de la sexualité pour découvrir de nouvelles potentialités moins centrées sur la pénétration comme performance. Rappelant que les féministes ont critiqué la réduction du domaine de l'érotisme et de la sexualité à la génitalité, l'autrice suggère qu'il convient de reconnaître que le désir de contact physique et de séduction est polymorphe et peut se recomposer tout au long du parcours de vie.

Face aux récits masculins centrés sur la perte de pouvoir sexuel, nombre d'autobiographies de femmes affirment au contraire le plaisir de se libérer de l'injonction à plaire et à désirer ${ }^{15}$. "Ne pas être désirée, c'est être libre " («To be unwanted is to be free ") écrit Germaine Greer et cette même idée est exprimée dans de nombreuses œuvres de femmes âgées explorées par Segal. Celle-ci se demande cependant si se détacher du désir sexuel et des passions charnelles constitue une forme de «libération » ou bien une façon de se "retirer du marché ", de devancer le regard social âgiste sur le corps féminin vieillissant, une forme de résignation face à une perte vue comme inévitable $d u$ pouvoir de séduire plutôt qu'un processus $d^{\prime}$ ' empowerment $»^{16}$. Sans répondre frontalement à cette question, le livre explore d'autres témoignages de femmes qui, au contraire, rendent compte d'un désir vivace en avançant en âge, mais aussi des transformations ou des bifurcations de ce désir. L'autrice évoque notamment les récits féminins - en incluant sa propre expérience - de découverte tardive de relations érotiques entre femmes après une longue trajectoire exclusivement hétérosexuelle et cite des œuvres littéraires et poétiques qui mettent en récit l'intimité et le désir entre personnes très âgées, quel que soit leur genre. À la fin du troisième chapitre intitulé « Les périls du désir », Segal confie : «Je lis les paroles de ces femmes et je réfléchis à ma propre vie. Je vois que je m'identifie à celles qui gardent les portes ouvertes à toutes sortes de passions en vieillissant. À la fin de la soixantaine, je pousse encore moi-même les portes, toujours en quête d'attention, d'affection et de louanges, avec la peur de les perdre un jour. Pourtant, même si je sais que je ne suis 
guère unique, je vois d'autres personnes s'arranger différemment avec le vieillissement. Je ne partage pas la tranquillité de celles qui se sentent libérées des périls du désir physique, ou qui disent ne plus craindre la perte de l'estime et de la considération sociale, mais [...] peut-être suivent-elles des chemins que je n'ai pas encore trouvés ». Le livre qu'elle publiera quatre ans après, Radical Happiness. Moments of Collective Joy, paru l'année d'émergence du mouvement \#Metoo, esquisse une réponse détournée à cette interrogation personnelle. Dans cet ouvrage, Segal explore notamment une joie spécifique qui survit avec l'avancée en âge, celle de se lier aux autres dans des luttes collectives, loin de l'assimilation individualiste du bonheur à une méthode de développement personnel, mais aussi loin des préjugés faisant de l'action collective et de la participation aux mobilisations pour le changement social un apanage de la jeunesse.

À partir d'une réflexion sur le désir et la sexualité en vieillissant, Segal en vient plus largement, on l'aura compris, à poser la question des " attachements", de ce qui nous lie aux autres et nous maintient au monde. Rappelant que la "dépendance» est constitutive de l'existence des humains en société à tous les âges, l'autrice critique l'opposition simpliste entre dépendance et autonomie qui organise les représentations dominantes de la vieillesse. Même si elle n'y fait pas directement référence, une partie de la littérature féministe sur le care met en question, de façon analogue, l'idée qu'il existerait des sujets autonomes, actifs, productifs et performants par opposition à d'autres sujets vulnérables, dépendants et improductifs. Au contraire, montre par exemple la politiste et théoricienne féministe Joan Tronto dans Un Monde vulnérable, la vulnérabilité et le care sont constitutifs de toute vie en société et "l'autonomie " apparente des uns existe précisément grâce à un travail de soutien, de sollicitude et de soin réalisé par d'autres ${ }^{17}$. Cette perspective critique fait écho dans Out of time à une double critique: d'une part celle des dimensions "classistes" de l'injonction à la performance et à l'autonomie (il est plus facile de s'y conformer en vieillissant quand on dispose de solides ressources économiques et que l'on n'a pas passé sa vie à exercer un métier pénible ${ }^{18}$; d autre part celle de de la distinction binaire entre un âge où l'on serait « actif » et engagé au monde, et un âge où l'on devrait s'en retirer et opter pour l'invisibilité, le détachement, la déprise et le retrait. Affirmant au contraire son désir de rester, autant que possible, engagée politiquement et affectivement - sans fuir la mélancolie, l'épreuve du deuil et la souffrance qui font partie de toute vie émotionnelle, particulièrement en avançant dans le grand âge -, Segal nous incite à explorer les conditions individuelles et collectives, psychiques et politiques susceptibles de contribuer à faire de la vieillesse une partie signifiante et significative de la vie.

\section{BIBLIOGRAPHIE}

Principaux ouvrages de Lynne Segal :

Segal, L. (1987) Is the Future Female? Troubled Thoughts on Contemporary Feminism, Virago Press. 
Segal, L. (1990) Slow Motion: Changing Masculinities, Virago Press; Rutgers Univ. Press, réed. augmentée et actualisée chez Palgrave, 2007.

Segal, L. (1994) Straight Sex: The Politics of Pleasure, Virago Press/California Univ. Press (Winner of Women and Psychogy Prize, USA).

Segal, L. (1999) Why Feminism? Gender, Psychology, Politics; Columbia Univ. Press.

Segal, L. (1997) New Sexual Agendas, London, McMillan, New York, New York Univ. Press.

Segal, L. (2007) Making Trouble: Life and Politics, London, Serpents Tail.

Segal, L. (2013) Out of Time:The Pleasures and Perils of Ageing, London and New York, Verso Books.

Segal, L. (2017) Radical Happiness: Moments of Collective Joy, London: Verso Books.

\section{NOTES}

1. Même s'il est dommage qu'il n'interroge pas les enjeux genrés de l'expression « OK boomer ! ", Guillaume LOHES défend de façon argumentée cette expression dans son texte « 'Ok Boomer' ou la déchirure d'une insoutenable vision du monde », Mediapart, 13 décembre 2019.

2. L'expression «boomer bashing» est notamment utilisée par Margaret Morganroth GULLETTE, Aged by culture, Chicago, University of Chicago Press, 2004, p. 44-46. Sur ce sujet voir aussi John MACNICOL, Neoliberalising old age, Cambridge, Cambridge University Press, 2015 et en français Jérôme PELLISSIER, La guerre des âges, Paris, Armand Colin, 2007.

3. Pour les années 1980-1990, on peut citer notamment Jo. ALEXANDER et al., Women and aging: an anthology by women, Corvallis, OR, Calyx Books, 1986 ; Sara ARBER et Jay GINN (dir.), Connecting gender and ageing: a sociological approach, Buckingham, Open Univ. Press, 1995; Barbara MACDONALD et Cynthia RICH, Look me in the eye: old women, aging, and ageism, San Francisco, Spinsters Book, Company, 1991 [1983]; Baba COPPER, Over the hill : reflections on ageism between women, Freedom, Calif, Crossing Press, 1988; Marilyn PeARsall, The other within us: feminist explorations of women and aging, Boulder (Colo.), Westview Press, 1997 ; Kathleen WoodWARD (dir.), Figuring Age : women, bodies, generations. Bloomington, Indiana University Press, 1999.

4. Margaret Morganroth G ULLETTE op.cit. voir aussi Susan P ICKARD, Age studies, London/Los Angeles, Sage, 2016.

5. V. p. 18. Ici et par la suite, la traduction en français des extraits cités de Out of Time est de mon fait.

6. Simone de BeAuvorr, Le Deuxième sexe, vol. II, Paris, Gallimard, Folio Essai (Chap. X, « De la maturité à la vieillesse », p. 456-482), 1999 [1949].

7. Simone de BeauvorR, La force des choses, Paris, Gallimard, NRF, 1963, p. 684-686.

8. Simone de Beauvorr, La vieillesse, Paris, Gallimard, NRF, 1970. Le livre est traduit en 1972 en anglais sous le titre The Coming of Age.

9. V. notamment Susan SONTAG, "The double standard of ageing ", The Saturday review, 23 sept. 1972, pp. 29-38.

10. Meg STACEY, "Older Women and Feminism: A note about my experience of the WLM", Feminist Review, n³1, 1989, pp. 140-142.

11. Sur ces débats, voir notamment Astrid HENRY, Not my Mother's Sister. Generational Conflict and Third-Wave Feminism. Bloomington \& Indianapolis, Indiana University Press, 2004 ainsi que Devoney Looser et Ann KAPLAN (dir.), Generations: Academic Feminists in Dialogue, Minneapolis, Univ. of Minnesota Press, 1997. 
12. Audre LORDE, «Age, Race, Class and Sex : Women redefining difference in Anne Mcclintock, Aamir Mufti and Ella Shohat, Dangerous Liaisons, gender, nation and postcolonial perspectives, Minneapolis, Minn : University of Minnesota Press, 1997, p.376

13. Sur cette question bien connue des psychanalystes, voir notamment André GREEN, Le temps éclaté, Paris, Éditions de Minuit, 2000.

14. Linn SANDBERG, Getting Intimate: A Feminist Analysis of Old Age, Masculinity and Sexuality, Linkoping, Linkoping University, 2011 (thèse de doctorat).

15. Sur les récits féminins du vieillissement, voir aussi Jeannette KING, Discourses of ageing in fiction and feminism : the invisible woman, New York, Palgrace Macmillan, 2013 et en français Martine BOyer-WeInMAnN, Vieillir, dit-elle: Une anthropologie littéraire de l'âge. Seyssel, Éditions Champ Vallon, 2013.

16. Dans son article «Ré-enchanter la vieillesse" (Mouvements $n^{\circ} 59,2009$, p. 113-122), la sociologue Rose-Marie LAGRAVE posait une question similaire à propos des femmes vieillissantes qui affirment ne plus s'intéresser à la sexualité.

17. Dans la préface à l'édition française Un Monde vulnérable. Pour une politique du care, Paris, La Découverte, 2009 (parution originale en anglais en 1993, trad. par Hervé Maury), Joan TRONTO écrit : « Nous sommes tous, à des degrés divers au cours de notre vie, tout comme au quotidien, destinataires de soins. Chacun de nous commence sa vie dans un état de complète dépendance et beaucoup d'entre nous feront à nouveau, d'une manière ou d'une autre, l'expérience de la dépendance au cours de leur vie; et nous ne cessons de prendre soin de nous-mêmes. Le care correspond ainsi à la fois à une capacité d'agir et à une passivité ; il implique que l'on soit acteur, mais aussi objet des actes des autres »(p.15). Voir aussi Patricia PAPERMAN et Sandra LAUGIER, Le souci des autres : éthique et politique du care, Paris, Éditions de l'EHESS « Raisons pratiques », 2005. 18. Sur ce point, voir aussi Stephen Katz, « growing older without aging? positive aging, antiageism and anti-aging » Generations, winter 2002, p.27-32

\section{RÉSUMÉS}

Dans quelle mesure le féminisme peut-il constituer une ressource pour vivre et penser autrement l'avancée en âge ? Cette interrogation constitue l'un des fils conducteurs de l'essai Out of Time de Lynne Segal. Chercheuse britannique en psychologie, études de genre et sexualité, Segal explore son propre rapport au vieillissement et à la vieillesse tout en s'appuyant sur des travaux sociologiques, psychanalytiques, historiques et littéraires. Cet article déplie et approfondit certaines des questions abordées par cet ouvrage en le faisant dialoguer avec d'autres écrits féministes sur le vieillissement et les relations entre générations.

To what extent can feminism be a resource for experiencing and thinking ageing differently? This question is one of the central threads of Out of Time, an essay in which Lynne Segal, a researcher specialising in gender studies, sexuality and psychology in the UK, explores her own relationship to ageing and old age in dialogue with a diversity of sociological, psychoanalytical, historical and literary works. This article unfolds and digs into some the issues addressed in this book in light of other feminist works on gender, ageing and intergenerational relations. 
INDEX

Mots-clés : âge, âgisme, baby-boom, Beauvoir (Simone de), féminisme, générations, genre, vieillesse, vieillissement

Keywords : age, ageing, ageism, baby boom, Beauvoir (Simone de), feminism, generations, gender.

\section{AUTEUR}

JULIETTE RENNES

Juliette Rennes, EHESS, CEMS 